\title{
Evaporation of ion-irradiated disks
}

\author{
C. P. Dullemond and H. C. Spruit
}

\author{
Max-Planck-Institut für Astrophysik, Karl-Schwarzschildstrasse 1, 85748 Garching, Germany \\ e-mail: dullemon@mpia.de
}

Received 10 December 2004 / Accepted 19 January 2005

\begin{abstract}
We calculate the evaporation of a cool accretion disk around a black hole due to the ion-bombardment by an ion supported accretion flow (here ISAF, or optically thin ADAF). As first suggested by Spruit \& Deufel (2002), this evaporation takes place in two stages: ion bombardment of the cool disk (Shakura-Sunyaev disk: SSD) produces an intermediate-temperature layer on top of the disk ("warm layer") which constitutes an independent accretion flow on both sides of the SSD. As this warm material accretes inward of the inner radius of the SSD, it becomes thermally unstable by lack of cooling of photons, and evaporates into the ISAF, thereby feeding the latter. Angular momentum conservation forces a certain fraction of the ISAF material to move outward, where it can bombard the SSD with its hot ions. The flow geometry is derived by computing stationary solutions of the continuity- and angular momentum equations for the three components (ISAF, warm flow and SSD). The overall radiative output is dominated by hard X-rays. They are produced mostly from the warm component, rather than the ISAF. The expected time dependence and stability of the flow, not computed here, is discussed briefly.
\end{abstract}

Key words. accretion, accretion disks - black hole physics - X-rays: binaries - galaxies: active

\section{Introduction}

The X-ray spectra of galactic black holes occur in two different "states": a soft state dominated by a soft multi-color blackbody component peaking around $1 \mathrm{keV}$, and a hard state in which the energy output peaks at $100-200 \mathrm{keV}$. The soft state can be explained well with a standard optically thick geometrically thin accretion disk of the kind of Shakura \& Sunyaev (1973). But what produces the emission in the hard state is still under debate. Some argue that it represents non-thermal synchrotron radiation from a jet (Markoff et al. 2001). Others invoke magnetic activity on the surface of the disk, creating a "corona" on top of the disk (Galeev et al. 1979; Haardt et al. 1994; Stern et al. 1995; Merloni \& Fabian 2001). A commonly cited explanation for the hard X-ray spectra of black holes is the comptonization of soft photons in of a hot optically thin virialized plasma flow. The concept of such a hot "ion torus" around black holes has already been proposed a long time ago (Shapiro et al. 1976; Ichimaru 1977; Rees et al. 1982), but optically thin plasmas in the $100 \mathrm{keV}$ range are notoriously unstable (Lightman \& Eardley 1974; Sunyaev \& Shakura 1975; Piran 1978; Chen et al. 1995). In the 90s it was recognized that the advection of thermal energy in an inefficiently radiating hot optically thin plasma flow can have a stabilizing effect on the flow (Narayan \& Yi 1995). Instead of converting potential energy into radiation, such an "Advection Dominated Accretion Flow" (ADAF, here called "Ion Supported Accretion Flow": ISAF) stores the released potential energy into thermal energy of the ions and simply advects it down through the event horizon. A crucial element in the theory of such ISAFs is the thermal decoupling of ions and electrons. If the temperature of the electrons would be equal to the (almost virial) temperature of the ions, the flow would cool dramatically and collapse. The weakness of the Coulomb coupling between the eletrons and the ions allows the electrons to have a much cooler temperature (of the order of $2 \times 10^{9} \mathrm{~K}$ ) than the ions (which can have temperatures reaching $10^{11} \mathrm{~K}$ ), and therefore to radiate only very limitedly, allowing the thermal energy to be advected into the black hole and the hot flow to be stable. Despite the relative inefficiency of ISAFs, they do emit at least some Bremsstrahlung and thermal synchrotron radiation, and they comptonize soft photons to high energies, which could explain the hard X-ray components observed in the spectra of black holes.

While this scenario seems appealing, there are a number of open issues. One of these is the existence of "intermediate" states in which both components are present simultaneously, suggesting that the hot ISAF and the cool SSD somehow coexist. Based on simple mean-free-path arguments one can show that if these flows exist at the same distance from the black hole, the ions of the ISAF (with energies of 1$100 \mathrm{MeV}$ ) are quickly lost by penetrating into the SSD, causing the ISAF to condense. Another issue is that ISAFs can only exist up to a certain distance from the black hole, because the two-temperature plasma state ceases to exist at low temperatures, (e.g. Igumenshchev et al. 1998; Czerny et al. 2000; Narayan \& Yi 1995). Therefore their existence means that they must have been produced in situ, probably by some evaporation mechanism acting on the cool Shakura-Sunyaev disk (hereafter "SSD"): the ISAF being the "vapor" from the SSD. 
A well-studied evaporation mechanism is "coronal" evaporation (which determines the chromosphere-corona transition in the solar atmosphere). It is governed by the conduction of heat by the electrons (Meyer \& Meyer-Hofmeister 1994). This has been shown to work well for models of Cataclysmic Variables. In the two-temperature regime, this process fails because the energy is not carried by the electrons any more. For black hole accretion the mechanism therefore only works well at large radii $\left(\gtrsim 10^{3} R_{S}\right)$. Yet there are strong indications that some evaporation mechanism acts on the SSD at much smaller radii: from analysis of X-ray spectra of the soft component it has been inferred that the inner radius of the SSD regularly moves outward from the last stable orbit to distances of a few tens of Schwarzschild radii, in particular during the transition from a "high-soft" state to a "low-hard" state (Zdziarski et al. 1999; Gilfanov et al. 2000; Churazov et al. 2001; Revnivtsev et al. 2001).

The simultaneous presence of a hot comptonizing plasma close to the black hole and an SSD with varying inner radius therefore poses a problem: what is the mechanism that evaporates the SSD at close distances from the event horizon, thereby producing the hot ISAF? Some time ago Spruit \& Deufel (2002) proposed that a possible mechanism could be in fact the injection of ISAF ions into the SSD. Their calculations show that such an ion bombardment produces an intermediatetemperature (about $60 \mathrm{keV}$ ) layer of optical depth $\tau \sim 1$ on top of the SSD surface (Deufel et al. 2002).

The temperature of the warm layer is kept stably in this intermediate range (which would be thermally unstable for an isolated optically thin plasma) by the balance between heating and Compton cooling by the soft photon flux from the cool disk. This is similar to the energy balance in the Haardt-Maraschi model (1994), but the present model is more predictive, since both the temperature and the optical depth of the layer are determined by the physics of ion penetration.

Such a "warm layer", being of much lower density and much higher temperature than the SSD (but still much lower than the virial temperature), transports its own angular momentum and forms an accretion flow on top of the disk. Now suppose the inner radius of the $\operatorname{SSD}\left(R_{\mathrm{ssd}}\right)$ is significantly far outside the last stable orbit. The radial flow velocity in the warm layer is orders of magnitude higher than the flow velocity in the SSD, and therefore this warm material quickly accretes inward of $R_{\mathrm{ssd}}$. In this region there is no SSD underneath the warm layer to provide soft photons for compton cooling, and therefore the temperature of the warm accretion flow inward of $R_{\mathrm{ssd}}$ increases (to roughly $300 \mathrm{keV}$, Deufel et al. 2002). Due to the reduced soft photon flux, this region is now also thermally unstable, and starts evaporating into the ISAF (Spruit \& Deufel 2002)

This process produces the mass flux that sustains the ISAF. Part of the ISAF material accretes inward, and part moves outward due to angular momentum conservation (as in any viscous disk, e.g. Pringle 1981; Liu et al. 1997; Turolla \& Dullemond 2000). The outward moving ISAF material spreads over the SSD. Over a short distance it dumps its ions onto the SSD, thus producing the warm layer and closing the cycle. This proposed mechanism is therefore a two-stage evaporation process: first

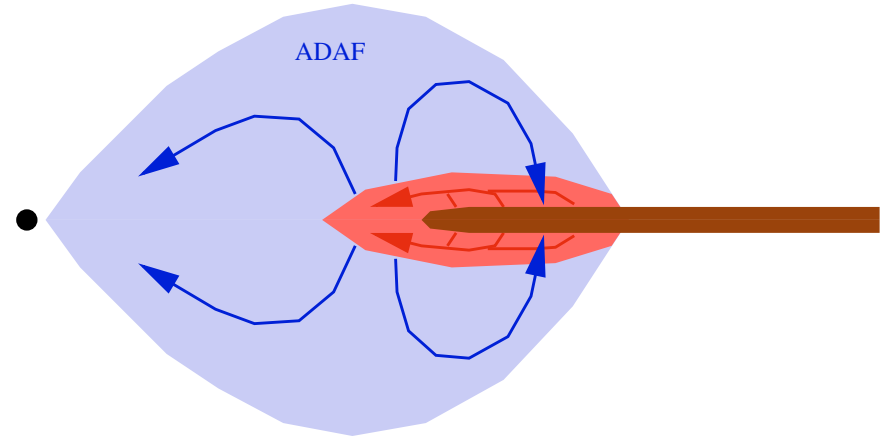

Fig. 1. The geometry of the three-layered accretion flow, in which evaporation of the cool disk takes place in two stages.

from the SSD into the warm layer, and then from the warm layer (at smaller radii) into the ISAF (see Fig. 1 for a sketch).

In the paper of Spruit \& Deufel "zero dimensional" estimates were given for the three-component flow geometry, without actual computation of the flow geometry. This is the topic of the present paper. We solve the combined continuity- and angular-momentum conservation equations for all three components (ISAF, warm flow and SSD) simultaneously with the appropriate mass exchange terms between them. We derive stationary solutions to these combined equations and study their properties.

\section{Basic model}

We treat all three components (ISAF, warm flow and SSD) in a vertically integrated way, but we allow the components to coexist with each other at every radius. Therefore, each component is represented by a surface density function $\Sigma_{\mathrm{x}}(R, t)$ where $R$ is distance from the center, $t$ is time and $\mathrm{x}$ stands for either "ISAF", "warm flow" or "SSD". Both the warm flow and the SSD may be truncated at an inner radius (larger than the last stable orbit). These radii, called $R_{\text {warm }}$ for the warm layer and $R_{\mathrm{ssd}}$ for the SSD may vary with time. In the stationary state they are eigenvalues of the problem and depend on the global accretion rate. The temperature of the ISAF is assumed to be a constant fraction times the local virial temperature:

$T_{\mathrm{isaf}}=1.26 \frac{\sqrt{1+0.56 \alpha^{2}}-1}{\alpha^{2}} \frac{G M}{R} \frac{\mu m_{\mathrm{p}}}{k}$

which can be derived from self-similar solutions of ADAFs using $\gamma=1.5$ (Narayan \& Yi 1994). The temperature of the SSD is calculated self-consistently assuming Kramer's opacity:

$$
T_{\mathrm{ssd}}(R)=1.17 \times 10^{8}\left(\alpha \Sigma_{\mathrm{ssd}}^{3} \frac{1}{\sqrt{\mu}} \frac{M / M_{\odot}}{R^{3}}\right)^{1 / 7} .
$$

The temperature of the warm layer is assumed to be $300 \mathrm{keV}$ for $R<R_{\mathrm{ssd}}$ and $60 \mathrm{keV}$ for $R \geq R_{\mathrm{ssd}}$. These values are estimates based on the results from Deufel et al. (2002), who calculated the detailed vertical structure of the warm flow. 


\subsection{Evaporation of warm layer into ISAF}

Spruit \& Deufel (2002) derived the evaporation rate of the warm layer inward of $R_{\mathrm{ssd}}$ into the ISAF. They find the following expression (valid for $R<R_{\mathrm{ssd}}$ ):

$\psi_{\text {warm } \rightarrow \text { isaf }}=\frac{m_{\mathrm{e}}^{3 / 2} k^{2}}{8 m_{\mathrm{e}}^{1 / 2} e^{4} \ln \Lambda} \alpha^{2} \Omega_{K}^{2} T_{\mathrm{e}}^{2}$,

(where $k T_{\mathrm{e}} \approx 300 \mathrm{keV}$ ). The evaporation rate is therefore constant between the inner radius of the warm layer and the inner radius of the cool disk. The inner radius of the warm layer $R_{\text {warm }}$ may be located at the last stable orbit at $R=$ $6 G M / c^{2}$, but it can also be larger if the evaporation process was so effective that it evaporates the layer before it has reached the black hole. At this stage, we assume the latter, i.e. that the warm layer has $R_{\text {warm }}>6 G M / c^{2}$. The consistency of this assumption will be part of the results obtained.

\subsection{Evaporation of SSD into warm layer}

The evaporation from the SSD into the warm layer for $R>R_{\mathrm{ssd}}$ is a more subtle matter. This evaporation is powered by the ion bombardment of the SSD by the ISAF. The flux of ions from the ISAF onto the SSD (and through the warm layer) is:

$\psi_{\text {isaf } \rightarrow \text { ssd }}=\xi \rho_{\text {isaf }} \sqrt{\frac{k T_{\text {isaf }}}{\mu m_{\mathrm{p}}}}$,

which is defined as the sum of the fluxes on both sides of the disk. The parameter $\xi$ is a dimensionless efficiency parameter of order unity. If $\xi=1$, the ISAF loses its matter at the fastest possible rate: any proton that reaches the disk surface is lost. Effects such as tangled magnetic fields or proton-proton collisions may reduce the loss rate of matter to the disk, which is simulated by taking $\xi<1$.

The protons that enter the SSD, leave their energy in the disk's upper layers. A fraction $\eta$ of this energy is radiated away by Bremsstrahlung and possibly other processes. It is not straightforward to compute this value $\eta$, so we keep it a parameter. The remainder of this energy can be used for the heating of disk matter up to the temperature of the warm layer. This creates an evaporation rate $\psi_{\mathrm{ssd} \rightarrow \text { warm }}$ of:

$\psi_{\text {ssd } \rightarrow \text { warm }}^{0}=(1-\eta) \psi_{\text {isaf } \rightarrow \text { ssd }} \frac{T_{\text {isaf }}-T_{\text {warm }}}{T_{\text {warm }}-T_{\text {ssd }}}$.

From the static solutions of Deufel et al. (2002) we know that, as $\Sigma$ approaches a value close to unity (let us define this value as $\Sigma_{0}$ ), the evaporation will cease and a static solution sets in. In this static solution the energy input by ion bombardment is entirely compensated by radiative losses (i.e. radiative losses in excess of the $\eta$ fraction already taken into account in the above evaporation rate formula). We simulate this saturation effect by multiplying $\psi_{\mathrm{ssd} \rightarrow \mathrm{ssd}}$ by a factor $\left(1-\Sigma_{\mathrm{warm}} / \Sigma_{0}\right)$. The mass gain of the warm layer is then:

$\psi_{\mathrm{ssd} \rightarrow \text { warm }}=\psi_{\mathrm{ssd} \rightarrow \text { warm }}^{0}\left(1-\frac{\Sigma_{\mathrm{warm}}}{\Sigma_{0}}\right)$.

Here, and in the remainder of this paper, we take $\Sigma_{0}=6 \mathrm{~g} / \mathrm{cm}^{2}$ (accounting for two sides of the Shakura-Sunyaev disk, each having a layer of maximum surface density of $3 \mathrm{~g} / \mathrm{cm}^{2}$ ).

\subsection{Equations for all three components}

All three layers (disk, warm layer and ISAF) obey their own equations of continuity and angular momentum conservation, with the appropriate sources terms to account for the exchange of matter between the them. The angular momentum exchange is given by the mass exchanged and the difference in specific angular momentum; viscous coupling between the layers is neglected. The continuity equation is best expressed as an equation for the local mass flux $\dot{M}_{\mathrm{x}}(R)$ (where x stands for either "ssd", "warm" or "isaf"). The equation for this is:

$\dot{M}_{\mathrm{x}}(R)=\dot{M}_{\mathrm{x}}\left(R_{0 \mathrm{x}}\right)-2 \pi \int_{R_{0 \mathrm{x}}}^{R} \psi_{\mathrm{x}}(R) R \mathrm{~d} R$,

where $R_{0 \mathrm{x}}$ is the inner edge of phase $\mathrm{x}$, which is $R_{\text {in }}$ for the ISAF, $R_{\text {warm }}$ for the warm layer and $R_{\text {ssd }}$ for the accretion disk. The quantity $\psi_{\mathrm{x}}$ is the mass source term. For the SSD one has $\psi_{\text {ssd }} \equiv \psi_{\text {isaf } \rightarrow \text { ssd }}-\psi_{\text {ssd } \rightarrow \text { warm }}$, for the warm layer one has $\psi_{\text {warm }} \equiv \psi_{\text {ssd } \rightarrow \text { warm }}-\psi_{\text {warm } \rightarrow \text { isaf }}$ and for the ISAF one has $\psi_{\text {isaf }} \equiv \psi_{\text {warm } \rightarrow \text { isaf }}-\psi_{\text {isaf } \rightarrow \text { ssd }}$.

The integration constant $\dot{M}_{\mathrm{x}}\left(R_{0 \mathrm{x}}\right)$ is zero for the warm layer and the SSD, since both have clearly defined inner edges due to evaporation. But it is non-zero for the ISAF, since this is the layer that in the end carries all the matter into the black hole. For the ISAF the value of $\dot{M}_{\text {isaf }}\left(R_{\text {in }}\right)$ is determined as an eigenvalue of the problem: by demanding a vanishing mass flux at the outer edge $\dot{M}_{\text {isaf }}\left(R_{\text {out }}\right)=0$, the value of $\dot{M}_{\text {isaf }}\left(R_{\text {in }}\right)$ is indirectly determined.

In the thin disk approximation the equation for angular momentum conservation with a no-friction boundary condition at $R=R_{0 \mathrm{x}}$ can be cast into the general form:

$\Sigma_{\mathrm{x}} v_{\mathrm{x}}=\frac{1}{6 \pi \sqrt{R}} \int_{R_{0 \mathrm{x}}}^{R} \frac{\dot{M}_{\mathrm{x}}(R)}{\sqrt{R}} \mathrm{~d} R$.

In order to solve the $\Sigma_{\mathrm{x}}$ from this, one must evaluate the viscosity coefficient $v_{\mathrm{x}}$, which for a standard $\alpha$-disk is given by

$v_{\mathrm{x}}=\alpha \sqrt{\frac{R^{3}}{G M}} \frac{k T_{\mathrm{x}}}{\mu m_{\mathrm{p}}}$.

\subsection{Numerical approach}

The above equations are solved in a set of nested subroutines and functions. At the deepest level we solve the structure of the ISAF for a given evaporation rate $\psi_{\text {warm } \rightarrow \text { isaf }}(R)$ and given $\dot{M}_{\text {isaf }}\left(R_{\text {in }}\right)$. This function returns the value of the ISAF mass flux at infinity $\dot{M}_{\text {isaf }}(R \rightarrow \infty)$. By feeding this function into a root-finding routine (we use zbrent from Numerical Recipes) the value of $\dot{M}_{\text {isaf }}\left(R_{\text {in }}\right)$ is found for which $\dot{M}_{\text {isaf }}(R \rightarrow$ $\infty)=0$. Note that this value is, due to recondensation of the outflowing part of the ISAF material, smaller than the total evaporation rate $2 \pi \int \psi_{\text {warm } \rightarrow \text { isaf }}(R) R \mathrm{~d} R$. In other words: some material goes more than once through the process of evaporation. In any case, the above procedure solves the ISAF structure for given $\psi_{\text {warm } \rightarrow \text { isaf }}(R)$.

One step higher in the hierarchy we solve the structure of the warm layer, for given values of $R_{\text {warm }}$ and $R_{\text {ssd }}$. First 
we integrate the equations from $R_{\text {warm }}$ to $R_{\text {ssd }}$ and determine $\psi_{\text {warm } \rightarrow \text { isaf }}(R)$. We then solve for the structure of the ISAF in the way described above. The ISAF spreads outward beyond $R_{\text {ssd }}$ and injects its protons into the SSD. This proton injection provides the energy needed to evaporate the SSD into the warm layer, and therefore determines the evaporation rate $\psi_{\mathrm{ssd} \rightarrow \text { warm }}$. This then enables us to continue the integration of the equations for the warm layer from $R_{\text {ssd }}$ out to $R \rightarrow \infty$.

The function that computes the structure of the warm layer in the above way returns the value of $\dot{M}_{\text {warm }}(R \rightarrow \infty)$. We feed this function into zbrent so that the value of $R_{\text {warm }}$ for which $\dot{M}_{\text {warm }}(R \rightarrow \infty)=0$ is automatically determined. Note that this is a nested use of zbrent, since within the function that determines the structure of the warm layer we also call zbrent for determining the structure of the ISAF.

\section{Resulting solutions}

Using the above procedure we find stationary solutions for most (though not all) parameter combinations. In Figs. 2-4 we show three examples, all of which have $M_{\mathrm{bh}}=10 M_{\odot}, \xi=1$ and $\eta=0.9$. In model 1 (Fig. 2) we take $\alpha=0.05$ for all three components, while in model 2 (Fig. 3) the ISAF and the warm layer have a ten times higher viscosity: $\alpha=0.5$. In both these models we take $R_{\text {ssd }}=30 R_{s}$. In model 3 we take $\alpha=0.5$ and $R_{\text {ssd }}=100 R_{s}$. As can be seen in these figures, the mass flux $\dot{M}$ for the ISAF is positive inward of a certain radius, and negative outward of that radius. This negative mass flux is the outflow in the ISAF driven by the angular momentum conservation. It rapidly goes back to zero toward larger radii due to the loss of ISAF ions into the SSD. The mass flux of the warm layer is zero at both small and large radii, and has a peak value at the inner edge of the SSD $\left(R_{\mathrm{ssd}}\right)$. Beyond $R_{\mathrm{ssd}}$ the mass of the SSD is evaporated into the warm layer, while at $R<R_{\mathrm{ssd}}$ the warm layer evaporates into the ISAF. The surface density of the warm layer makes a jump at $R_{\text {ssd }}$, which is due to the temperature jump (300 keV inward of $R_{\text {ssd }}$ and $80 \mathrm{keV}$ outward of $R_{\text {ssd }}$ ). In the case of low viscosity (model 1, Fig. 2) the warm layer only narrowly reaches the saturation surface density $\Sigma_{\text {warm }}=\Sigma_{0} \equiv 6.0$, but for the high viscosity case (model 2, Fig. 3) this saturation value is reached easily. For the same high $\alpha$ but with larger $R_{\mathrm{ssd}}$, the surface density does not reach saturation anymore.

Interestingly, this saturation of $\Sigma_{\text {warm }}$ causes the mass flux in the SSD to acquire an peculiar wiggle in model 2 (clearly seen in Fig. 3): first (at large radii) the matter in the SSD is evaporated into the warm layer $\left(\mathrm{d} \dot{M}_{\mathrm{ssd}} / \mathrm{d} R>0\right)$, but as one gets closer to the inner radius $R_{\mathrm{ssd}}$, some matter of the warm layer is dumped back onto the SSD $\left(\mathrm{d} \dot{M}_{\mathrm{ssd}} / \mathrm{d} R<0\right)$. Only just beyond $R_{\text {ssd }}$ the evaporation of the SSD kicks in again and produces the cut-off in $\dot{M}_{\text {ssd }}$ and the maximum in $\dot{M}_{\text {warm }}$ at $R_{\text {ssd }}$. This recondensation takes place because if the surface density of the warm layer has reached the saturation value $\Sigma_{0}$ (which is independent of radius), the mass flux in the warm layer should go roughly proportional to $R^{3 / 2}$ because both $\Sigma_{\text {warm }}$ and the temperature of the warm layer are then constant with $R$. A decreasing mass flux in the warm layer in the flow direction $\left(\mathrm{d} \dot{M}_{\text {warm }} / \mathrm{d} R<0\right)$ inevitably means recondensation onto the
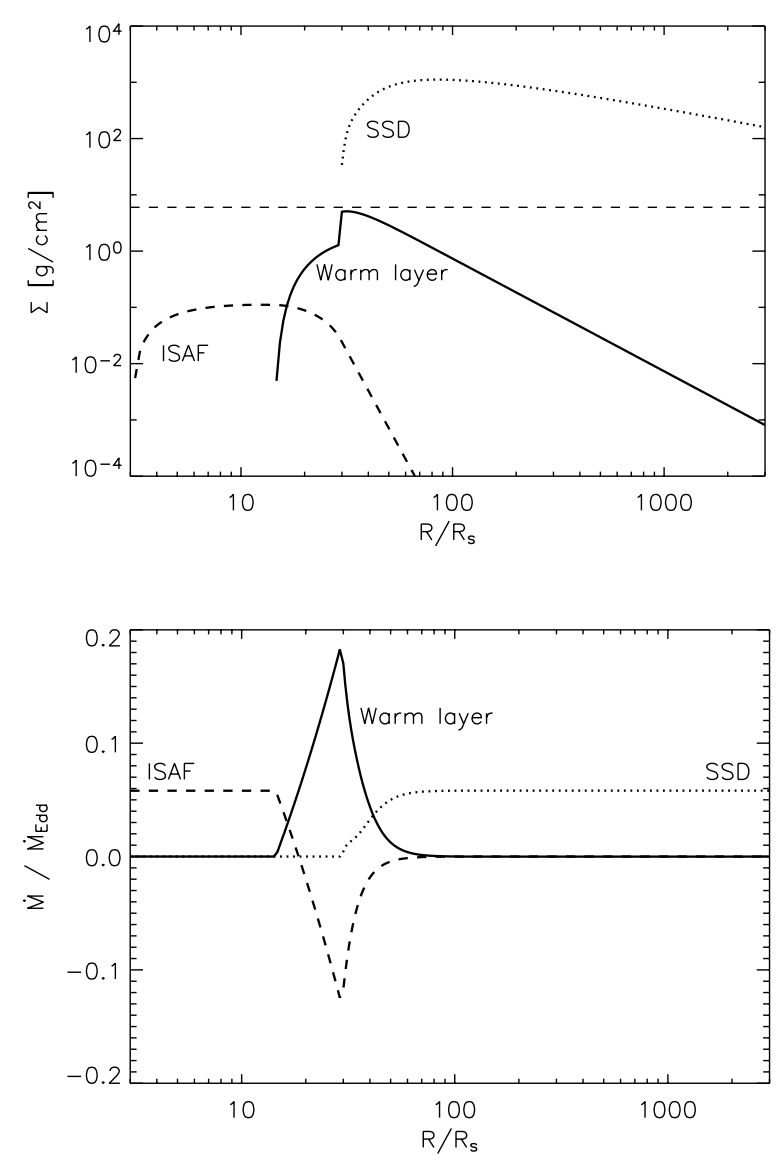

Fig. 2. The radial three-layered structure of the evaporating disk and the hot accretion flows, for the case of low $\alpha$ (i.e. $\alpha=0.05$ ) for the warm layer and the ISAF (model 1). Upper panel shows the surface density of the two components: the cool (SSD) disk, warm surface layer and the ISAF. Lower panel shows the local mass flux of the components in units of the Eddington rate (the Eddington accretion rate is defined here using an efficiency factor of 0.1 ). The horizontal dashed line in the top figure is the value of $\Sigma_{0}=6 \mathrm{~g} / \mathrm{cm}^{2}$.

SSD. This wiggle is not present in the case of low viscosity or the case with large $R_{\mathrm{ssd}}$, since the saturation value $\Sigma_{0}$ is not reached by the warm layer.

Another interesting difference between the example models is that the $300 \mathrm{keV}$ part of the warm layer (i.e. the part inward of $R_{\mathrm{ssd}}$ ) is narrower for the case of high viscosity (model 1) than for the case of low viscosity (model 2).

\subsection{Global trends}

The dependence of some global parameters of the models on the input parameter $R_{\mathrm{ssd}}$ are discussed in this subsection. In Fig. 5 the width of the $300 \mathrm{keV}$ part of the warm layer (i.e. the part inward of $R_{\mathrm{ssd}}$ ) is shown as a function of $R_{\mathrm{ssd}}$. It is clear that for low $\alpha$ and for large $R_{\mathrm{ssd}}$ this width is the largest. Since this region may be responsible for the production of hard X-rays, its surface area is an important quantity of the model. Also, if this region is wide, then the approximation that the soft photons of the SSD do not cool this region (hence allowing it to have $300 \mathrm{keV}$ temperature) is more valid than in the very narrow case. 

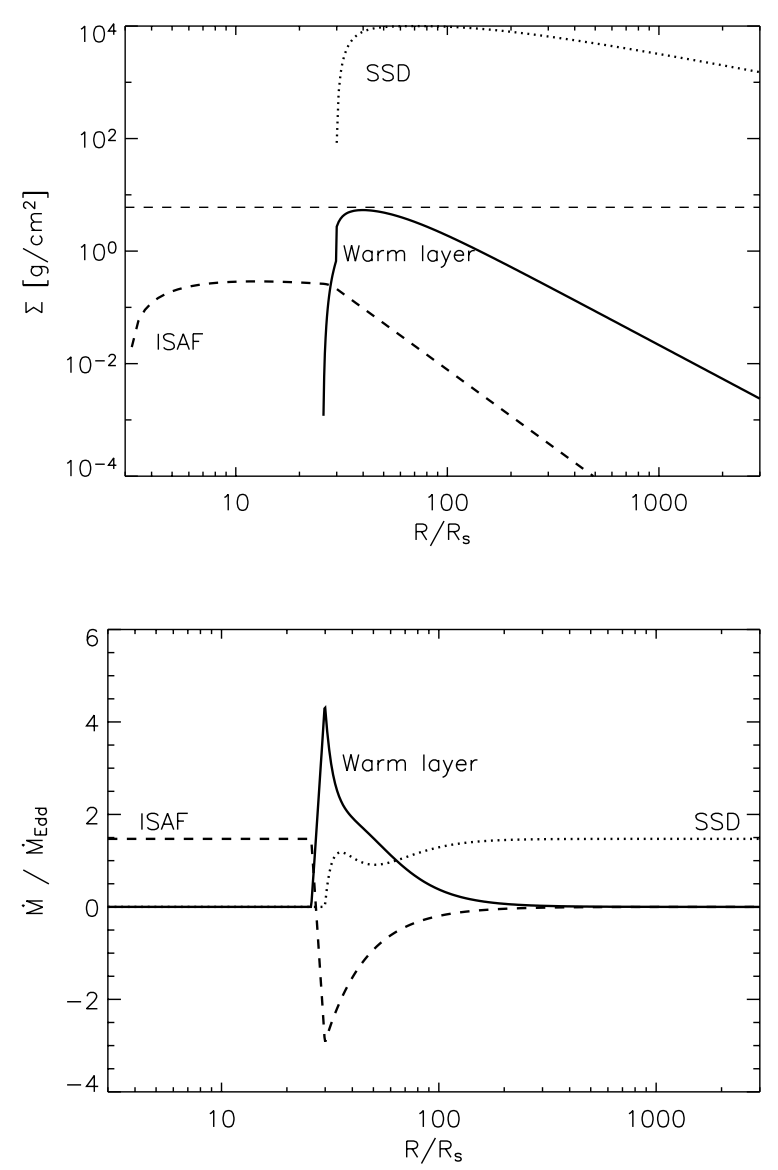

Fig. 3. The radial structure for the case of $\alpha=0.5$ (model 2).

In Fig. 6 the circulation rate is shown. It tells how often the matter is evaporated and recondensed again before accreting onto the hole. As one can see, for all values of $\alpha$ and $R_{\text {ssd }}$ this rate is always of the order of 3 , meaning that on average the ISAF matter gets injected into the SSD (and re-evaporated from the warm layer) twice before finally flowing into the black hole. In other words: $2 / 3$ of the hot ISAF matter is used up for the proton injection into the SSD.

\subsection{Radiative output}

The three components of the flow each contribute to the X-ray spectrum. The cool disk produces a very soft component, the warm layer and the ISAF a hard component (peaking above $100 \mathrm{keV}$ ). A detailed calculation of the overall spectrum produced by the combination of these components would be somewhat premature, in view of the uncertainties in the model. The relative contributions of hard and soft X-rays, however, can be determined simply from the energy budgets of these components. Figure 7 shows these in the form of the vertically integrated heating rates of the components, per unit surface area. The ISAF has the largest amplitude, since the energy release in it takes place in the deepest part of the gravitational potential. The radiative efficiency of an ISAF is low, however, so that the radiative output is actually dominated by the other two components of the flow, in particular the warm component. The exact value of the radiative efficiency of an ISAF
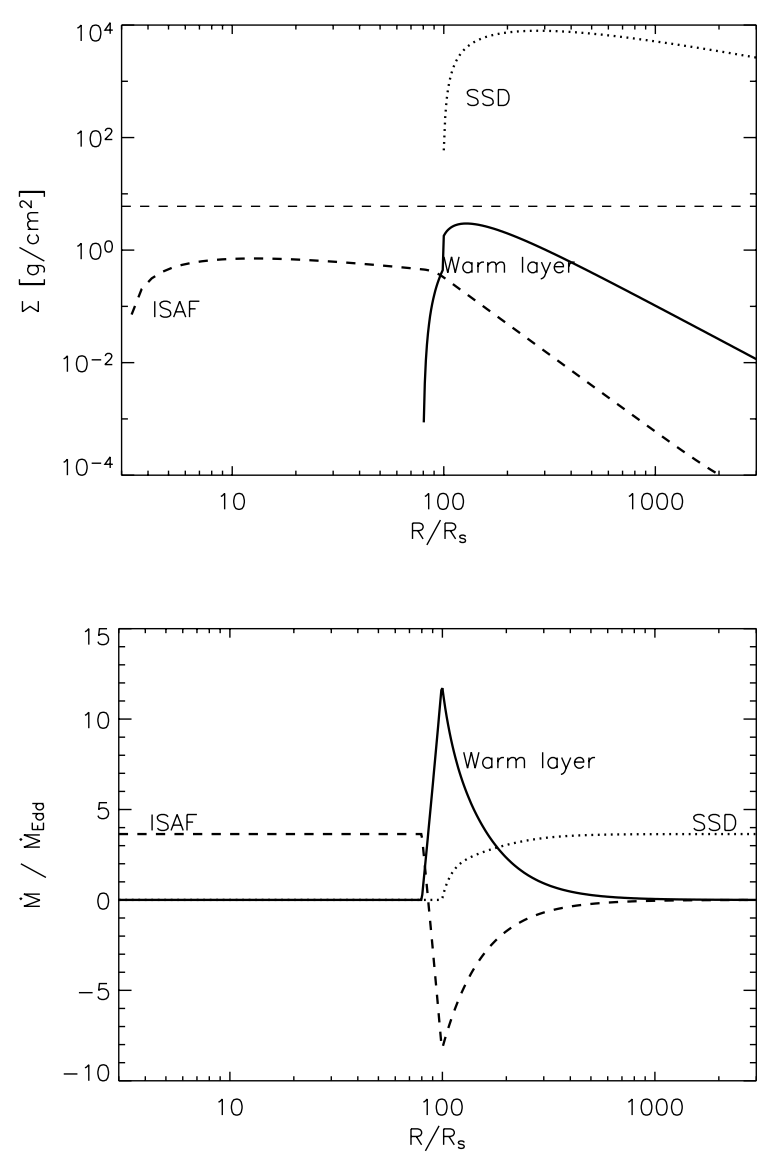

Fig. 4. The radial structure for the case of $\alpha=0.5$ (like model 2) but this time with $R_{\mathrm{sdd}}=100 R_{s}(\operatorname{model} 3)$.

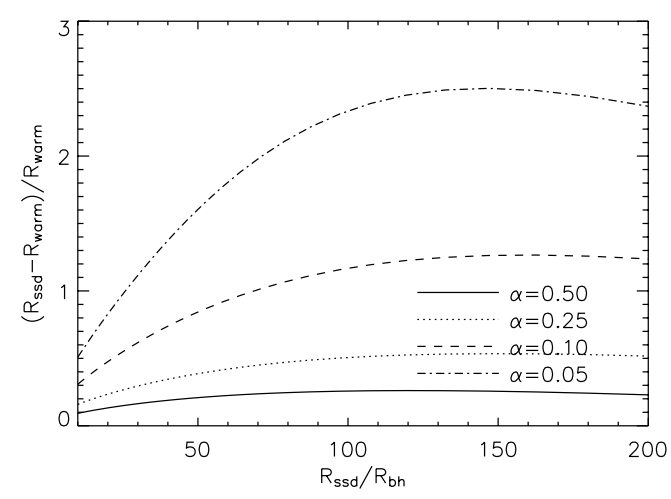

Fig. 5. The width of the $300 \mathrm{keV}$ region of the warm layer as a function of $R_{\mathrm{ssd}}$. This width is defined as $R_{\mathrm{ssd}} / R_{\mathrm{warm}}-1$.

depends on details, but typical numbers are of the order $\epsilon \approx$ $\dot{m} \alpha^{2}$ (e.g. Rees et al. 1982; Esin et al. 1997). For the case shown in Fig. 7 , this yields $\epsilon \approx 1.5 \times 10^{-4}$. The contribution of the ISAF is thus small, and most hard radiation comes from the warm layer. The total energy deposition into the warm layer (viscous heating plus ion illumination) is $Q_{\text {tot,warm }}=$ $1.2 \times 10^{35} \mathrm{erg} / \mathrm{s}$ for the example of Fig. 7 (model 1). All of this goes into hard radiation, but a fraction of this radiation is intercepted by the cool disk and reprocessed into soft flux. The internal viscous dissipation in the cool disk is a smaller 


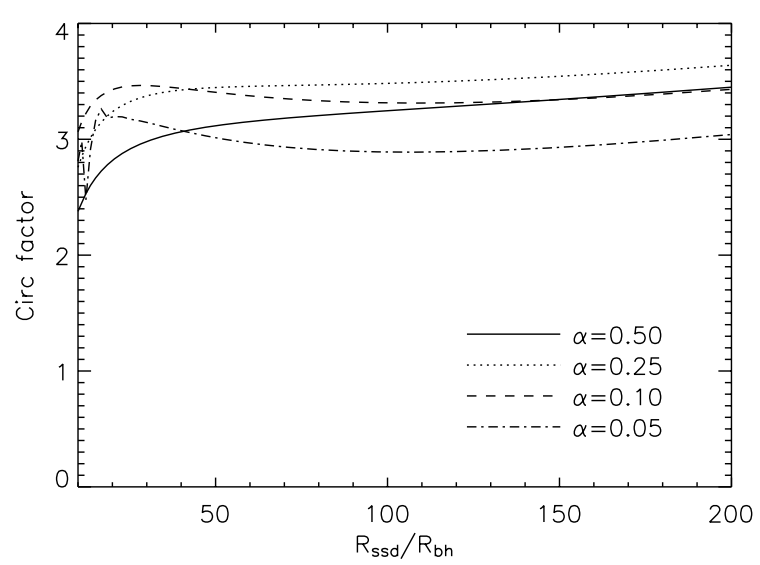

Fig. 6. The circulation rate within the stationary solution.

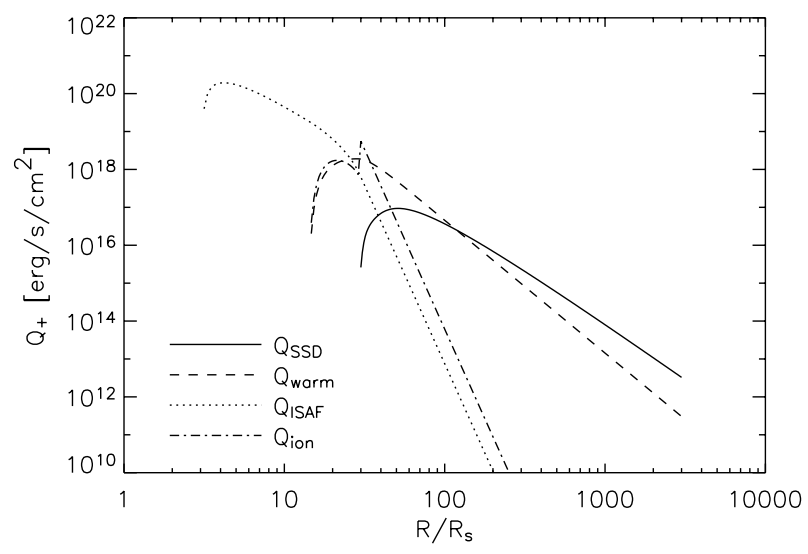

Fig. 7. Energetics of the three components of the accretion flow for model 1 (i.e. $\alpha=0.05$ for all components). Solid: radiation from the cool disk, dashed: viscous dissipation in the warm component, dotdashed: heating of the warm layer by ion illumination from the ISAF, dotted: viscous dissipation in the ISAF. Since the ISAF is radiatively inefficient, the total radiative output from the flow is dominated by the hard radiation from the warm layer. The accretion rate for this case is $\dot{m}=0.06$ (in units of Eddington).

contribution $\left(Q_{\mathrm{tot}, \mathrm{ssd}}=4.6 \times 10^{34} \mathrm{erg} / \mathrm{s}\right)$. In this way, we find, for the example of Fig. $7, F_{\text {hard }} / F_{\text {soft }} \approx 2.6$. The cool disk produces a standard multi-color disk spectrum; since its inner edge is at some distance from the hole, its contribution to the X-ray spectrum is very soft. Most of the energy dissipated in the warm layer is converted to to hard X-rays by Comptonization of soft photons from the cool disk (a fraction is spent in poducing soft flux by irradiation of the cool disk surface). The ISAF also produces a hard spectrum, but since it is radiatively inefficient, its contribution to the total hard flux is much smaller than its energy budget as shown in Fig. 7.

The conclusion is thus that the model produces a predominantly hard spectrum. The soft flux produced is mostly due to reprocessing of hard flux by the cool disk directly underneath the warm layer, but includes a small contribution from internal viscous heating in the disk. The hard flux comes mostly from the region where the warm layer evaporates into the ISAF, i.e. a ring just inside the inner edge of the cool disk. This is consistent with the close connection found observationally between the timing properties of the hard flux and the inner radius of the cool disk (e.g. Gilfanov et al. 2000; Churazov et al. 2001; Revnivtsev et al. 2001).

\subsection{Stability}

\subsubsection{Stability of the vertical structure}

If we envisage the radial structure of the cool disk as fixed, one can study whether the structures of the warm and hot (ISAF) layers is stable to small perturbations. This question was answered in part by Deufel et al. (2002). In these calculations, the vertical structure of a cool disk plus warm layer under ion illumination was determined in a time dependent way, but without the radial structure that we have included here. In these calculations, the results quickly converge to a stable static equilibrium, as long as the cool disk is present. The adjustment takes place on the thermal time scale of the warm layer. This stability can be understood as a consequence of the overall energy balance (Haardt et al. 1994) between comptonization in the warm layer and soft photon production in the cool disk. Inside the inner edge of the cool disk, however, the warm layer becomes unstable due to the lack of soft photon flux, and transforms into the evaporating flow studied here.

\subsubsection{Stability of the radial structure}

In the present calculations, additional questions of stability arise related to the radial structure of the accretion flows. The first question we posed is if the combination of ISAF, warm layer and cool disk is stable if the position of the inner edge of the cool disk is kept fixed. In other words, if the evaporation into the ISAF, its spreading over the cool disk and condensation onto it converges to a stable stationary state. Preliminary timedependent simulations of the warm disk, for a fixed accretion disk, and for a stationary ISAF computed at every time-step, show that the warm layer seems to be stable, and will find the stationary solution quickly. But full time dependent simulations of both hot phases (the warm disk and the ISAF) have not been carried out to confirm this.

A second question is whether the position of the inner edge of the cool disk is stable: on time scales longer than the adjustment time of the warm layer, the balance between evaporation and condensation may lead to a drift of the position of the inner edge of the cool disk. This question can be answered already without a full time dependent study by considering the relation between the radius of the inner edge of the accretion disk and the total mass accretion rate. This is shown in Fig. 8. It shows that for increasing accretion rate the inner radius of the cool disk (as well as the inner radius of the warm layer) increases as well. The reason is that in our model the warm flow near $R_{\mathrm{ssd}}$ must carry the entire mass accretion rate $\dot{M}$ while it's surface density is limited to at most $\Sigma_{0}$. For higher $\dot{M}$ it therefore requires a larger circumference to transfer all the matter with the same surface density, hence the larger $R_{\text {ssd }}$.

This relation is opposite to what is found from the coronal evaporation mechanism of Meyer \& Meyer-Hofmeister (1994), where the inner radius of the cool disk decreases for increasing global accretion rate. This relation caused the 


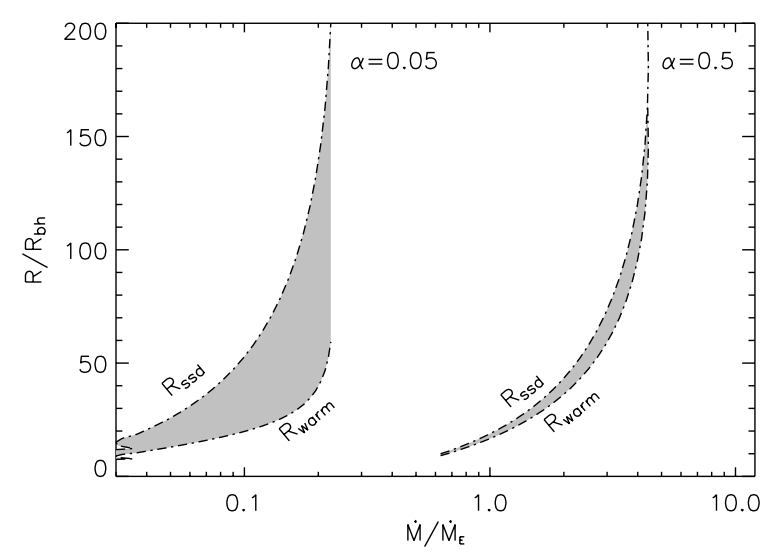

Fig. 8. The inner radius of the cool disk $R_{\mathrm{ssd}}$ and the inner radius of the warm phase $R_{\text {warm }}$ as a function of global accretion rate $\dot{M}$ in units of the Eddington accretion rate $\dot{M}_{\mathrm{E}}$ (defined using a $10 \%$ efficiency factor). The grey area shows therefore the extend of the $300 \mathrm{keV}$ evaporating inner part of the warm layer inward of the cool disk. Shown are the results for models $1(\alpha=0.05)$ and $2(\alpha=0.5)$. Note that in the modeling procedure the $R_{\text {ssd }}$ is the model parameter and $\dot{M}$ the result. But since in nature it is the opposite, we plot $\dot{M}$ on the $x$-axis here.

Meyer \& Meyer-Hofmeister evaporation mechanism to be selfregulating: if the $R_{\text {ssd }}$ is too large, it will decrease until a balance is reached between the total mass accretion rate in the disk and the evaporation rate. Such a self-regulatory mechanism is not present in ion-bombardment evaporation process. A small deviation from the stationary solution may let the $R_{\mathrm{ssd}}$ move e.g. slightly inward, which decreases the allowed global accretion rate for a stationary solution. Since the accretion rate is fixed from the outside and cannot be changed, it will exceed this new value and therefore decrease $R_{\text {ssd }}$ even further. We therefore expect that a small deviation of $R_{\mathrm{ssd}}$ from the equilibrium value would amplify and cause the inner edge of the SSD to move inwards or outwards in a run-away fashion. The time scale to be expected for this instability is the viscous time scale of the SSD.

The question thus arises whether the evaporating solutions found here can survive in practice; in any case it is clear that some additional piece of physics will be needed. This need not be seen as a debilitating disadvantage, however, since the variability of the hard state shows that the accretion flow is in fact violently unstable. If the evaporation mechanism had predicted a stable stationary flow, it would have needed additional physics as well, in this case something to explain the observed instability of the flow.

\section{Discussion and conclusions}

In this paper we have presented the structure of the 3-layered accretion disk model envisaged by Spruit \& Deufel (2002). This models is based on the ion-illumination model for X-ray binaries in which a hot ion supported flow coexists with a cool accretion disk (Deufel et al. 2002; Spruit and Deufel 2002). This coexistence leads to a three-layered structure: between the cool disk and the ion supported flow a "warm" layer is formed by ions penetrating the disk from the ion supported flow. The ion and electron and electron temperatures are in equilibrium in this warm layer (in contrast with ion supported flow, where the ion temperature is much higher than the lectron temperatue). Its temperature, in the $100 \mathrm{keV}$ range, is governed by the equilibrium between ion heating and Compton cooling by soft photons from the cool disk. This equilibrium is quite stable, in contrast with a single optically thin accretion flows in this temperature range, which are strongly thermally unstable (the well known instability of the "SLE" branch of accretion solutions, e.g. Chen et al. 1995). In the paper of Deufel et al. (2002) the local vertical structure (for a given distance from the hole) of the accretion flow was calculated. The calculations presented here are complementary to these. They focus instead on the radial structure of the accretion flow, while the vertical structure is approximated by a 3-layer approximation. The exchange of energy and angular momentum between these layers is taken into account, as well as the processes of evaporation and condensation between the ion supported flow and the warm layer, and between the warm layer and the cool disk.

We find that stationary solutions exist for a wide range of parameters, and that these flow geometries exhibit circulation patterns in which matter is evaporated and recondensed several times before vanishing into the black hole. This finding is of special significance for the Li-enhancements observed in the secondaries of black hole binaries (Martín et al. 1994), which can be explained as a result of Li-production in the accretion disk by ion illumination (spallation of CNO nuclei and $\mathrm{He}+\mathrm{He}$ fusion), provided that in addition to the accretion flow there is an outflow from the inner regions of the accretion disk that can carry the Li to the secondary. This is an attractive possibility since the hard spectral states that we associate here with the ion-illumination process is known to be closely related to the presence of jets in in black hole binaries. The "recirculation" of matter through the ion-bombarded warm layer increases the Li-production significantly.

We find that the energy radiated by the ion-illuminated, evaporating flow is likely to be dominated by a hard X-ray component produced in the evaporating region itself. A soft component is also produced by reprocessing in the cool disk, but it is energetically less important than the hard component. This provides a satisfactory solution to a long-standing problem in models where the hard and soft fluxes are coupled through reprocessing. The generic model for this coupling (Haardt \& Maraschi) has the property of predicting similar energy fluxes in the hard and soft components. In order to make the hard component dominate as observed, modifications of the geometry are needed to reduce the interaction between the soft photons and the Comptonizing region. In the evaporating flows presented here, this occurs naturally because most of the hard flux is produced in a "soft photon staved" region just inside the inner edge of the cool disk.

We also show that the globally stationary solutions are not stable on long time scales. Eventually the inner rim of the evaporating SSD will move either outward or inward in a run-away fashion. However, if the inner rim of the SSD is kept fixed, the structures of the warm intermediate layer and the hot ISAF are presumably stable. Hence, this evaporation mechanism is feasible, but it will result in time-dependent phenomena instead of 
a stationary state. How these time-dependent solutions behave remains the topic of a future detailed time-dependent study of ion-illuminated evaporating disks.

\section{References}

Chen, X., Abramowicz, M. A., Lasota, J.-P., Narayan, R., \& Yi, I. 1995, ApJ, 443, L61

Churazov, E., Gilfanov, M., \& Revnivtsev, M. 2001, MNRAS, 321, 759

Czerny, B., Rozycska, A., Janiuk, A., \& Zycki, P. T. 2000, New Astron. Rev., 44, 439

Deufel, B., Dullemond, C. P., \& Spruit, H. C. 2002, A\&A, 387, 907

Esin, A. A., McClintock, J. E., \& Narayan, R. 1997, ApJ, 489, 865

Galeev, A. A., Rosner, R., \& Vaiana, G. S. 1979, ApJ, 229, 318

Gilfanov, M., Churazov, E., \& Revnivtsev, M. 2000, MNRAS, 316, 923

Haardt, F., Maraschi, L., \& Ghisellini, G. 1994, ApJ, 432, L95

Ichimaru, S. 1977, ApJ, 214, 840

Igumenshchev, I. V., Abramowicz, M. A., \& Novikov, I. 1998, MNRAS, 298, 1069

Lightman, A. P., \& Eardley, D. M. 1974, ApJ, 187, L1
Liu, B. F., Meyer, F., \& Meyer-Hofmeister, E. 1997, A\&A, 328, 247

Markoff, S., Falcke, H., \& Fender, R. 2001, A\&A, 372, L25

Martin, E. L., Rebolo, R., Casares, J., \& Charles, P. A. 1994, ApJ, 435, 791

Merloni, A., \& Fabian, A. C. 2001, MNRAS, 328, 958

Meyer, F., \& Meyer-Hofmeister, E. 1994, A\&A, 288, 175

Narayan, R., \& Yi, I. 1994, ApJ, 428, L13

Narayan, R., \& Yi, I. 1995, ApJ, 452, 710

Piran, T. 1978, ApJ, 221, 652

Pringle, J. E. 1981, ARA\&A, 19, 137

Rees, M. J., Begelman, M. C., Blandford, R. D., \& Phinney, E. S. 1982, Nature, 295, 17

Revnivtsev, M., Gilfanov, M., \& Churazov, E. 2001, A\&A, 380, 520

Shakura, N. I., \& Sunyaev, R. A. 1973, A\&A, 24, 337

Shapiro, S. L., Lightman, A. P., \& Eardley, D. M. 1976, ApJ, 204, 187

Siuniaev, R. A., \& Shakura, N. I. 1975, Soviet Astron. Lett., 1, 158

Spruit, H. C., \& Deufel, B. 2002, A\&A, 387, 918

Stern, B. E., Poutanen, J., Svensson, R., Sikora, M., \& Begelman, M. C. 1995, ApJ, 449, L13

Turolla, R., \& Dullemond, C. P. 2000, ApJ, 531, L49

Zdziarski, A. A., Lubinski, P., \& Smith, D. A. 1999, MNRAS, 303, L11 